\title{
The active RS CVn-type system SZ Pictoris
}

\author{
C. I. Martínez, ${ }^{1,2 \star ~ J . ~ F . ~ G o n z a ́ l e z, ~}{ }^{2}$ A. Buccino, ${ }^{3,4}$ R. Ibañez Bustos ${ }^{3}$ \\ and P. J. D. Mauas ${ }^{3,4}$ \\ ${ }^{1}$ Observatorio Astronómico Félix Aguilar, Universidad Nacional de San Juan, Av. Benavides 8175 oeste, CP 5400 San Juan, Argentina \\ ${ }^{2}$ Instituto de Ciencias Astronómicas de la Tierra y el Espacio (CONICET-Universidad Nacional de San Juan), Av. España 1512 sur, San Juan, Argentina \\ ${ }^{3}$ Instituto de Astronomía y Física del Espacio (CONICET-UBA), C.C. 67 Sucursal 28, C1428EHA Buenos Aires, Argentina \\ ${ }^{4}$ Departamento de Física, Facultad de Ciencias Exactas y Naturales, UBA, CP 1428 Buenos Aires, Argentina
}

Accepted 2019 October 23. Received 2019 October 7; in original form 2019 July 1

\begin{abstract}
We study the short-term variability and long-term variability of the spectroscopic binary SZ Pictoris, a southern RS CVn-type system. We used mid-resolution Echelle spectra obtained at Complejo Astronómico El Leoncito spanning $18 \mathrm{yr}$, and the photometric data from the All Sky Automated Survey data base ( $V$ band) and from the Optical Robotic Observatory (BVRI bands) for similar time lapses. We separated the composite spectra into those corresponding to both components, and we were able to determine accurate orbital parameters, in particular an orbital period of $4.95 \mathrm{~d}$. We also observed a photometric modulation with half the orbital period, due to the ellipticity of the stars. We also found cyclic activity with a period of $\sim 2030 \mathrm{~d}$, both in the photometry and in the Ca II flux of the secondary star of the system.
\end{abstract}

Key words: stars: activity - stars: fundamental parameters - stars: individual: SZ Pictoris.

\section{INTRODUCTION}

RS CVn stars are close detached binaries formed by a giant or subgiant star of spectral types $\mathrm{G}-\mathrm{K}$ as the more massive primary component and a subgiant or dwarf star of spectral types $\mathrm{G}-\mathrm{M}$ as the secondary. Since these stars are fast rotators tidally synchronized, they show higher levels of activity than single stars with the same characteristics. In particular, these systems typically exhibit strong Ca II, H $\alpha$, X-ray, and microwave emissions (Hall 1976). At optical wavelengths, the most prominent feature of the RS CVn stars is their periodic photometric variability, which is thought to be the consequence of rotational modulation by large dark star-spots (see, for example, Roettenbacher et al. 2016). On the other hand, many of these stars show long-term chromospheric activity variations and activity cycles (Buccino \& Mauas 2009).

SZ Pictoris is a southern star reported as a double-lined spectroscopic binary and photometric variable of the RS CVn type by Andersen, Nordström \& Olsen (1980). They found that both components have similar spectral types and exhibit $\mathrm{Ca}$ II $\mathrm{H}$ and $\mathrm{K}$ line-core emission. The apparent magnitude is $V=7.893 \mathrm{mag}$, and the spectral line ratios correspond to a luminosity ratio of $0.7-0.8$ (Andersen et al. 1980). The effective temperature $T_{\text {eff }}=5310 \mathrm{~K}$ and the lithium abundance $\log n(\mathrm{Li})<1.8$ were determined by Pallavicini, Randich \& Giampapa (1992) using high-resolution $(R=50.000)$ and high-S/N $(\geq 100)$ spectra.
Cutispoto (1998a) determined a spectral type K0/1 IV + G5 IV for the system. However, he later found that higher luminosities were required to fit the Hipparcos distance (172-224 pc; Turon et al. 1992) and he adopted a K0 IV/III + G3 IV/III (Cutispoto 1998b). The more precise distance obtained by the Gaia mission $(d=188.4 \pm 0.9 \mathrm{pc}$; Gaia Collaboration 2018) confirms this luminosity-class assignment.

Rocha-Pinto \& Maciel (1998) obtained an activity level for the system of $\log R_{\mathrm{HK}}^{\prime}=-4.05$ and a difference between photometric and spectroscopic metallicity of $\Delta[\mathrm{Fe} / \mathrm{H}]=-0.444$. Later, RochaPinto et al. (2000) suggested that the low photometric metallicity can be explained as an effect of the reddening, since at SZ Pic's distance we should expect that the object is mildly to strongly reddened, resulting in a photometric metallicity that would seem lower than it really is. They calculated the reddening in the region of SZ Pic using $\beta$ index from Hauck \& Mermilliod (1998), the intrinsic colour calibration of Schuster \& Nissen (1989), and the Hipparcos distance $194.9 \mathrm{pc}$, obtaining $E(b-y)=0.064$.

Using a method based on the concept of common chromospheres (roundchroms), Gurzadyan (1997b) determined an intercomponent distance of $14.9 \mathrm{R}_{\odot}$, as well as an electron concentration in the roundchroms of $n_{\mathrm{e}}=7 \times 10^{10} \mathrm{~cm}^{-3}$. This method supposed a roundchrom enveloping both components of the binary system without coming into contact with their photospheres. It is based on the identification of the outer boundary of a roundchrom with an equipotential zero-velocity surface at some value of the Jacobi constant $\mathrm{C}$, at which the formation of a narrow corridor near the central Lagrangian point $L_{2}$ will be ensured for the transit of gaseous matter from the main component of the system in the direction of 
Table 1. Logs of the CASLEO observations of SZ Pic. Columns 1 and 3: date of each observing run (MMYY). Columns 2 and 4: heliocentric Julian date $\mathrm{xJD}=\mathrm{HJD}-2450000$.

\begin{tabular}{llll}
\hline Label & xJD & Label & xJD \\
\hline 0800 & 1770 & 1206 & 4081 \\
0301 & 1973 & 1208 & 4821 \\
0302 & 2364 & 0309 & 4904 \\
0802 & 2520 & 0310 & 5264 \\
1102 & 2601 & 0311 & 5638 \\
0303 & 2715 & 1212 & 6282 \\
0903 & 2898 & 1212 & 6285 \\
1203 & 2981 & 0313 & 6356 \\
0304 & 3074 & 1013 & 6592 \\
0904 & 3277 & 0314 & 6736 \\
1104 & 3336 & 1214 & 7007 \\
0305 & 3449 & 1118 & 8436 \\
1105 & 3700 & 1118 & 8437 \\
\hline
\end{tabular}

the secondary component. The central concept of the determination of the radius of the main component of the system is based on the assumption that the observed $\mathrm{Mg}$ II emission $(\lambda=2800 \AA)$ is entirely of roundchrom origin (Gurzadyan 1997a).

In 1999, we started the HK $\alpha$ Project to study the long-term chromospheric activity in late-type stars using medium-resolution spectra obtained at the Complejo Astronómico El Leoncito (CASLEO), San Juan, Argentina. In the framework of this project, for SZ Pic, we measured the activity index $S_{\mathrm{Mw}}=0.54$ (Cincunegui, Díaz \& Mauas 2007b) contrasting with the earlier observation by Henry et al. (1996), who obtained $S_{\mathrm{MW}}=0.975$. In this work, we present spectra of SZ Pic spanning more than $18 \mathrm{yr}$, together with two photometric data sets obtained by the All Sky Automated Survey (ASAS; Pojmanski 2002) and our own observations. In Section 2, we present an overview of our spectroscopic and photometric observations and the reduction methods employed. In Section 3, we describe the method used to study the temporal evolution of the data. In Section 4, we describe the results and, finally, we discuss the results and possible future work in Section 5.

\section{OBSERVATIONS AND DATA REDUCTION}

Since 2000, we have been continuously observing SZ Pic as part of the ongoing $\mathrm{HK} \alpha$ Project. This data set allows us to have a unique long time series of Mount Wilson indexes, which is the most extended activity indicator used to detect stellar activity cycles (Baliunas et al. 1995; Cincunegui, Díaz \& Mauas 2007a; Metcalfe et al. 2013; Flores et al. 2017). The observations were obtained with the REOSC spectrograph $^{1}(R=\lambda / \Delta \lambda \approx 13.000)$, mounted on the $2.15 \mathrm{~m}$ JS telescope at the CASLEO. This equipment provides us with mid-resolution Echelle spectra covering a wavelength range between 3800 and $6900 \AA$, which allows us to simultaneously study the effect of chromospheric activity in the whole optical spectrum. These Echelle spectra were optimally extracted and flux calibrated as explained in Cincunegui \& Mauas (2004). The resulting spectral resolution is high enough to clearly resolve the double features in the spectra of SZ Pic at appropriate phases.

Table 1 lists the spectral observation logs of SZ Pic. The first and third columns show the date (month and year as MMYY) of the observations and the second and fourth columns list $\mathrm{xJD}=\mathrm{HJD}-$

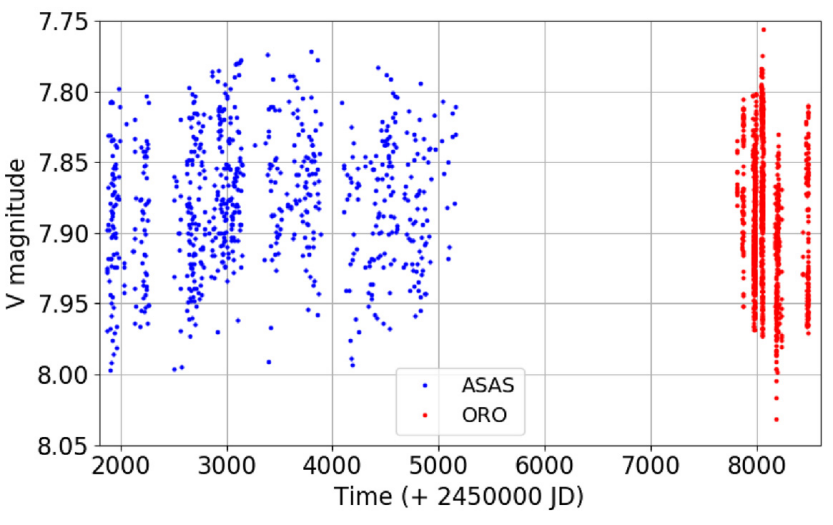

Figure 1. $V$ magnitude of SZ Pic as a function of time (ASAS in blue; ORO in red).

2450000, where HJD is the heliocentric Julian date at the beginning of the observation. There is a total of 26 individual observations distributed from 2000 August to 2018 November.

We also carried out photometric observations of SZ Pic using the four 16 arcsec MEADE telescopes that form the Optical Robotic Observatory (ORO) installed at the Observatorio Felix Aguilar (OAFA), also located in San Juan, Argentina. We observed SZ Pic for 4-5h per night in non-consecutive nights to have a nearuniform sampling throughout the expected orbital phase. During each observing night, we obtained science images every 5 min approximately. In total, we obtained 797 good images between 2018 March and 2019 January. We estimated the formal error for each observation as the sum in quadrature of the errors of the fluxes of the target and the reference star. The maximum errors were near 17 mmag.

In addition, we also employed photometric observations provided by the ASAS-3 data base. We only used observations qualified as A or B in the data base ('best' or 'mean' quality, respectively, according to the ASAS definition). The final time series consists of 734 points, between 2000 November and 2009 November, with typical errors of around $31 \mathrm{mmag}$. In Fig. 1, we plot the $V$ magnitude of SZ Pic as a function of time, obtained with ASAS-3 and ORO. Similarly to our previous works (Díaz et al. 2007; Buccino et al. 2011, 2014; Ibañez et al. 2019), we chose the mean $V$ magnitude of each observing season as a proxy for magnetic activity.

\section{ORBITAL PARAMETERS OF THE SYSTEM}

In Fig. 2, we show an example of the original spectra, corresponding to $2001 \mathrm{March}$, in the $\mathrm{Ca}$ II region. The double emission peaks can be easily noted. We processed these original spectra using the iterative method developed by González \& Levato (2006). This method allows us to separate the individual spectra of each component, and to compute their radial velocities (RVs). In each iteration, the spectrum computed for one component is removed from the observed one. The resulting single-lined spectrum is then used to measure the RV of the remaining component and to compute its spectrum by combining them appropriately.

We employed the mid-resolution Göttingen Spectra Libraries by Phoenix ${ }^{2}$ (Husser et al. 2013) to select the template for the crosscorrelations, according to the spectral classification by Cutispoto 


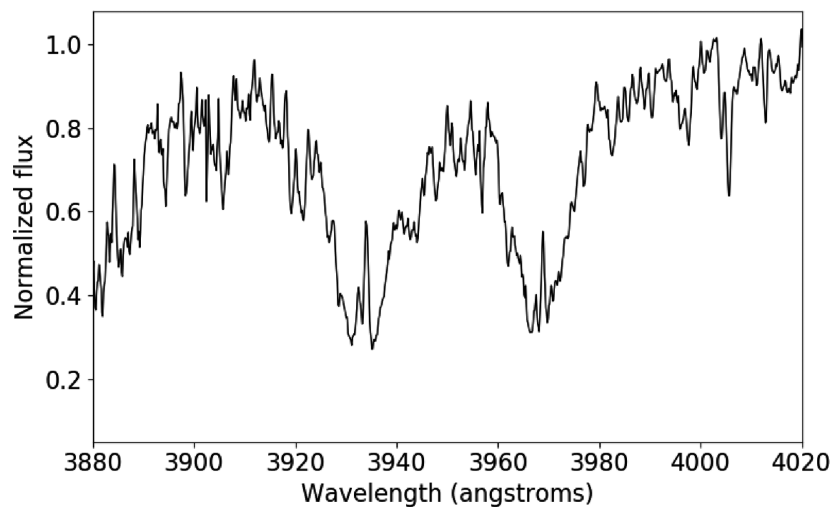

Figure 2. Example of an original composite spectrum in the Ca II region.

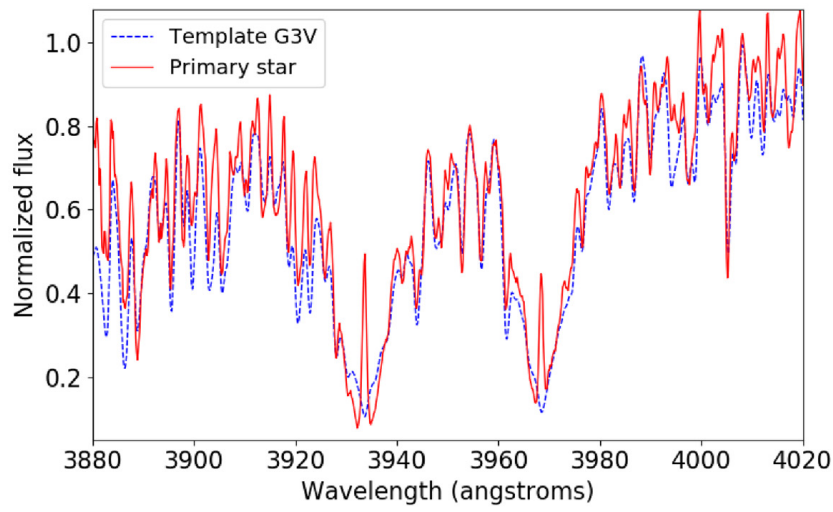

(a)

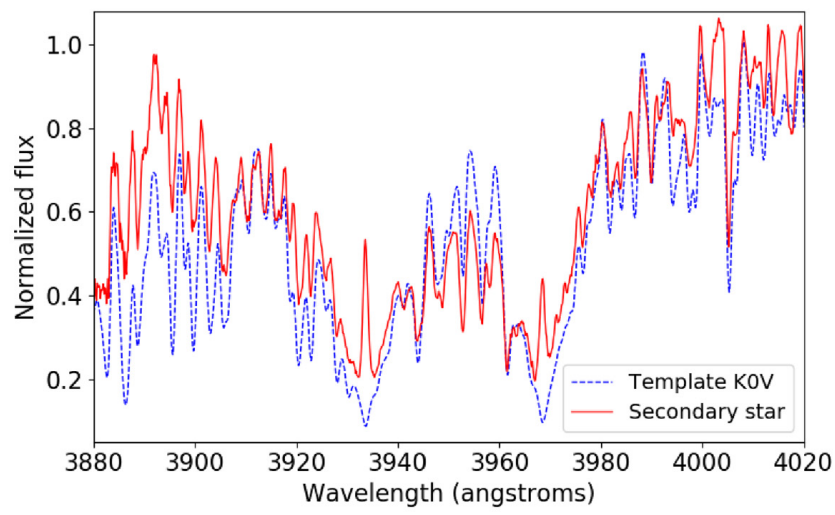

(b)

Figure 3. Mean spectra in the Ca II region of each component (red solid line) compared with the corresponding templates (blue dashed line).

(1998b) mentioned in Section 1. In all cases, for the RV measurements, we excluded the emission lines. A mean spectrum of very high $\mathrm{S} / \mathrm{N}(\geq 100)$ was obtained for each component. In Fig. 3, we show both spectra compared with the templates in the $\mathrm{Ca}$ II $\mathrm{H}$ and $\mathrm{K}$ region.

The Lomb-Scargle (LS) periodogram (Horne \& Baliunas 1986) has been extensively employed to search for stellar activity cycles (e.g. Baliunas et al. 1995; Metcalfe et al. 2013; Flores et al. 2017). Recently, Zechmeister \& Kürster (2009) proposed a modification, the generalized Lomb-Scargle (GLS) periodogram, which has certain advantages in comparison to the classic LS periodogram: It takes into account a varying zero-point, it does not require a

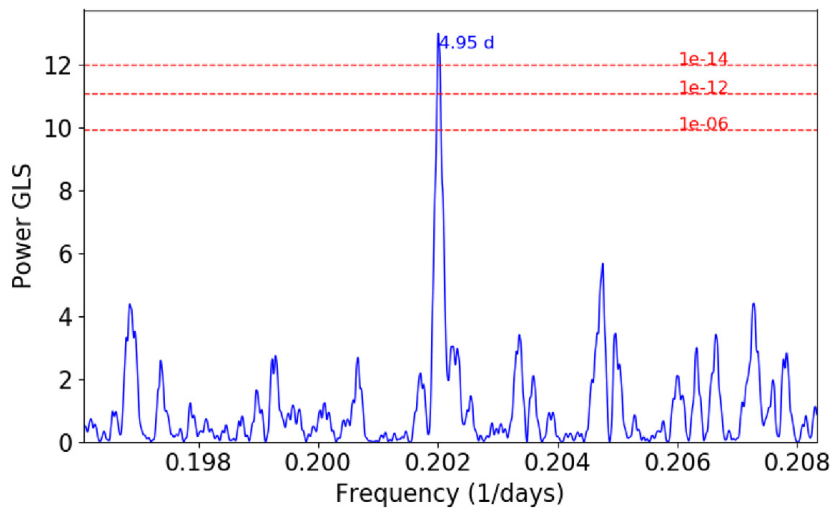

Figure 4. GLS periodogram of the differential RVs between the primary and secondary components. The most significant peak is indicated. The dashed lines indicate the false alarm probability levels.

bootstrap or Monte Carlo algorithms to compute the significance of a signal, reducing the computational cost, and it is less susceptible to aliasing than the LS periodogram.

We calculated the GLS periodogram to the RV difference between the components, to eliminate the possible systematic errors or eventual variations of the systemic velocity (see Fig. 4). The amplitude of the periodogram at each frequency point is identical to the value that would be obtained estimating the harmonic content of a data set, at a given frequency, by linear least-squares fitting to a harmonic function of time (Press et al. 1992).

To estimate the false alarm probabilities (FAPs) of each peak of the periodogram, we use

$F A P=1-\left[1-\left(1-\frac{2 P}{N-1}\right)^{(N-3) / 2}\right]^{M}$,

where $N$ is the size of the data set, $M$ is the number of independent frequencies, and $P$ is the power of the period detected in the GLS periodogram (Zechmeister \& Kürster 2009). We normalize the periodogram assuming Gaussian noise.

We obtained an orbital period $P=4.950320 \pm 0.000014 \mathrm{~d}$, with an FAP smaller than $10^{-14}$. Using this period, we fit a Keplerian orbit to the RVs of the system (see Fig. 5). The orbital parameters are listed in Table 2.

By comparing the mean spectra of the two components with synthetic spectra from the Göttingen Spectra Library of Husser et al. (2013) based on Phoenix atmosphere models, we estimated the effective temperatures and flux ratio between the components, obtaining: $T_{\text {eff }}(A)=5700 \pm 300 \mathrm{~K}, T_{\text {eff }}(B)=5400 \pm 300 \mathrm{~K}$ and $f_{A} / f_{B}=2.24 \pm 0.33$. Additionally, we computed the absolute magnitude using the apparent magnitude and the Gaia distance $(d=188.4 \pm 0.9 \mathrm{pc})$, obtaining $M_{\mathrm{V}}=1.46 \pm 0.10 \mathrm{mag}$ for the whole system. In these calculations, the interstellar extinction $\left(A_{\mathrm{V}}=0.074 \mathrm{mag}\right)$ was calculated from the Schlegel, Finkbeiner \& Davis (1998) maps and the distance, applying the same procedure as Bilir et al. (2008).

Then, using the flux ratio and appropriate bolometric corrections (Flower 1996), we estimate the absolute bolometric magnitude for each component: $2.64 \pm 0.16$ mag for primary star and $1.70 \pm 0.12$ for secondary star. Finally, we derive the stellar radius of the components from their luminosities and temperatures: $2.7 \pm 0.4 \mathrm{R}_{\odot}$ for primary and $4.6 \pm 0.5 R_{\odot}$ for secondary. We found no evidence of eclipses in the photometry, therefore, we can set an upper limit for the inclination angle. From the spectroscopic parameter 


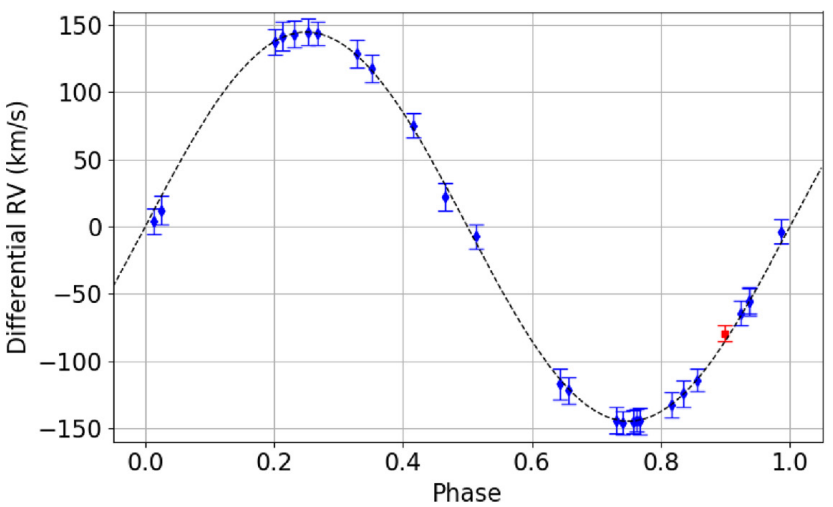

(a)

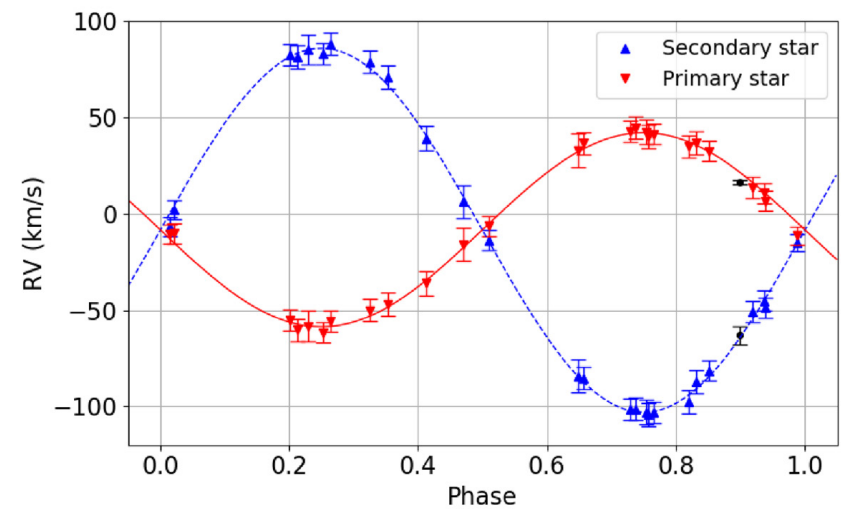

(b)

Figure 5. RV measurements of each component; the black points correspond to individual RVs determined by Andersen et al. (1980), $16.2 \pm 1.2$ and $-63.1 \pm 4.5 \mathrm{~km} \mathrm{~s}^{-1}$ in JD $=2443789.8801$ (1978 October). In both the figures, the dashed lines are the best fits to the data.

Table 2. Orbital parameters for SZ Pic.

\begin{tabular}{lr}
\hline Conjunction epoch (HJD) & $24511111.1903 \pm 0.0059$ \\
Period (days) & $4.950320 \pm 0.000014$ \\
$a \sin i\left(\mathrm{R}_{\odot}\right)$ & $14.14 \pm 0.14$ \\
Systemic velocity $\left(\mathrm{km} \mathrm{s}^{-1}\right)$ & $-8.47 \pm 0.21$ \\
Primary $\mathrm{RV}$ amplitude $\left(\mathrm{km} \mathrm{s}^{-1}\right)$ & $50.36 \pm 0.78$ \\
Secondary RV amplitude $\left(\mathrm{km} \mathrm{s}^{-1}\right)$ & $94.40 \pm 0.69$ \\
Orbital eccentricity & $0.016 \pm 0.007$ \\
$M_{1} \sin ^{3} i\left(\mathrm{M}_{\odot}\right)$ & $1.014 \pm 0.015$ \\
$M_{2} \sin ^{3} i\left(\mathrm{M}_{\odot}\right)$ & $0.54 \pm 0.011$
\end{tabular}

Table 3. Intrinsic properties of SZ Pic.

\begin{tabular}{lcc}
\hline & Primary star & Secondary star \\
\hline$T_{\text {eff }}(\mathrm{K})$ & $5700 \pm 300$ & $5400 \pm 300$ \\
$L\left(\mathrm{~L}_{\odot}\right)$ & $6.5 \pm 1.0$ & $15.4 \pm 1.8$ \\
$R\left(\mathrm{R}_{\odot}\right)$ & $2.7 \pm 0.4$ & $4,6 \pm 0.5$ \\
$M\left(\mathrm{M}_{\odot}\right)$ & $>1.54$ & $>0.82$ \\
\hline
\end{tabular}

$a \sin i=14.14 \mathrm{R}_{\odot}$ and the estimated radii, it can be inferred that the inclination angle $i$ is lower than $63 \mathrm{deg}$. In turn, this imposes lower limits for the stellar masses: $M_{1}>1.54$ and $M_{2}>0.82 \mathrm{M}_{\odot}$. In Table 3, we summarize the intrinsic properties of the components of SZ Pictoris.
Table 4. GLS analysis of the different photometry data sets.

\begin{tabular}{lccc}
\hline Data & $\begin{array}{c}\text { Photometric period } \\
\text { (days) }\end{array}$ & $\begin{array}{c}\text { Amplitude } \\
\text { (days) }\end{array}$ & $\begin{array}{c}\text { Magnitude } \\
(\mathrm{mag})\end{array}$ \\
\hline$B$ band (ORO) & $2.4758 \pm 0.0004$ & $0.0501 \pm 0.0016$ & $8.6590 \pm 0.0011$ \\
$V$ band (ORO) & $2.4755 \pm 0.0002$ & $0.0534 \pm 0.0011$ & $7.8931 \pm 0.0008$ \\
$R$ band (ORO) & $2.4756 \pm 0.0003$ & $0.0572 \pm 0.0022$ & $7.7328 \pm 0.0015$ \\
$I$ band (ORO) & $2.4759 \pm 0.0004$ & $0.0525 \pm 0.0017$ & $7.4161 \pm 0.0012$ \\
$A S A S-3$ & $2.4752 \pm 0.0001$ & $0.0472 \pm 0.0018$ & $7.8777 \pm 0.0011$ \\
\hline
\end{tabular}

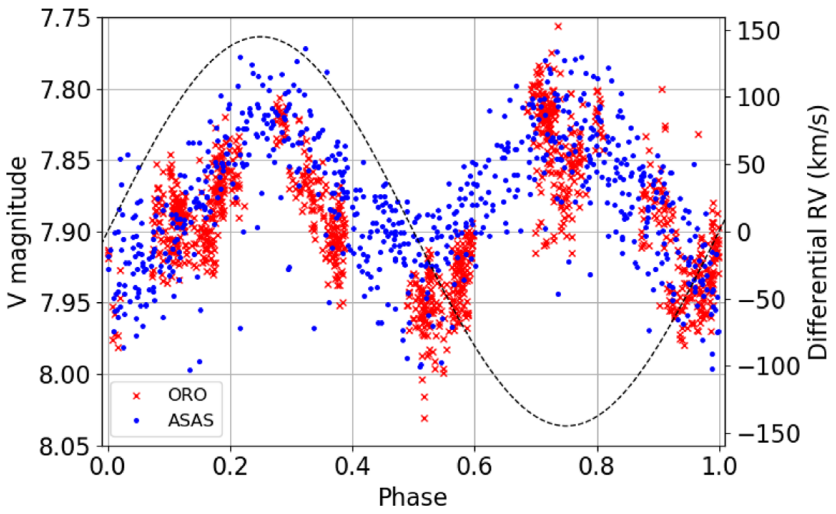

Figure 6. Photometric variation for ASAS (blue) and ORO (red) together with the fit to the rotation (black dashed line).

To search for rotational modulations in the photometric data, we applied the GLS periodogram to the photometric observations described in Section 2. In Table 4, we summarize the results obtained for the $B-, V-, R$-, and $I$-band data observed with ORO, and the $V$ observations from ASAS. We see that the photometric periods obtained agree very well, and are exactly half the orbital period, with errors smaller than 0.03 per cent and very low FAP levels. The results are shown in Fig. 6, where we show the photometry phased to the orbital period together with the Keplerian orbit. It can be seen that the system is brighter when the velocity is maxima, i.e. at quadrature. This result agrees with the hypothesis formulated by Drake, Simon \& Linsky (1989) that the photometric period for these synchronized systems should be half the orbital period, and that most of the variability is caused by an ellipticity effect on the shape of the stars (Cutispoto 1998b).

Bell, Hall \& Marcialis (1983) estimated a photometric period of $P_{\text {phot }}=2.441 \mathrm{~d}$ with an amplitude of the light curve of about $0.15 \mathrm{mag}$, using the data set of Andersen et al. (1980). Later, Strassmeier et al. (1993) found an orbital period $P_{\text {orb }}=4.92$ d, and Cutispoto (1995) obtained a photometric period $P_{\text {phot }}=4.905 \mathrm{~d}$. All these values agree very well with our results.

\section{STELLAR ACTIVITY}

To search for a long period, associated with a stellar activity cycle, we first removed from the complete photometry the photometric period $P=2.47 \mathrm{~d}$ found in Fig. 4, since it is too strong and masks any other variation. Then, we computed the GLS periodogram to the residuals. As can be seen in Fig. 7, there are two distinct peaks: The most significant peak corresponds to a period of $P=2030 \pm 34 \mathrm{~d}$ and the second peak corresponds to a period of $P=3167 \pm 166 \mathrm{~d}$. For both the peaks, the FAP is lower than $1 \times 10^{-14}$. However, the length of the ASAS data set is of $3289 \mathrm{~d}$. Therefore, we discard 


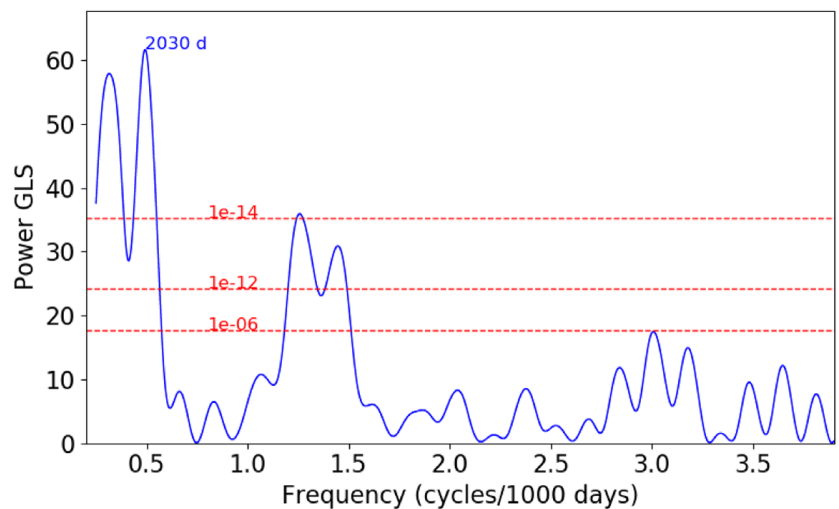

(a)

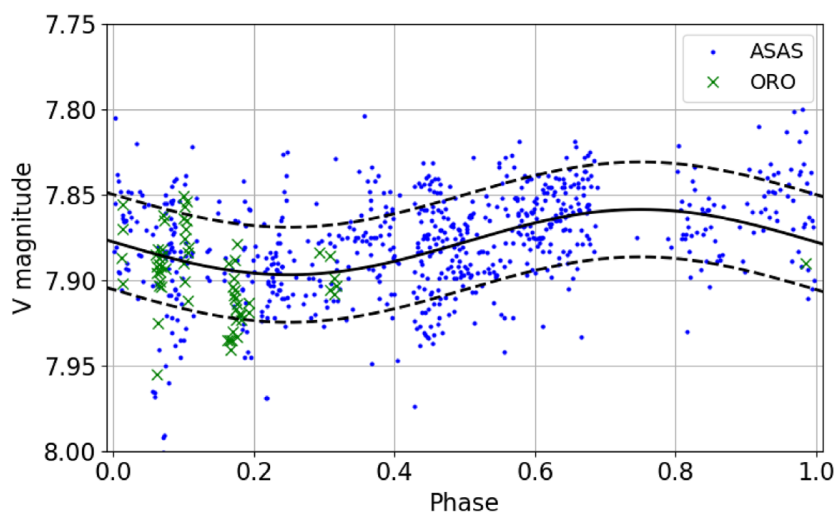

(b)

Figure 7. (a) GLS periodogram of the complete photometry data set; the most significant peaks are indicated; the dashed lines indicate the FAP levels. (b) Phase curve for $P=2030 \mathrm{~d}$; the ORO photometry is represented by the mean magnitude (green markers) and the dashed lines represent $\pm \sigma$ deviations.

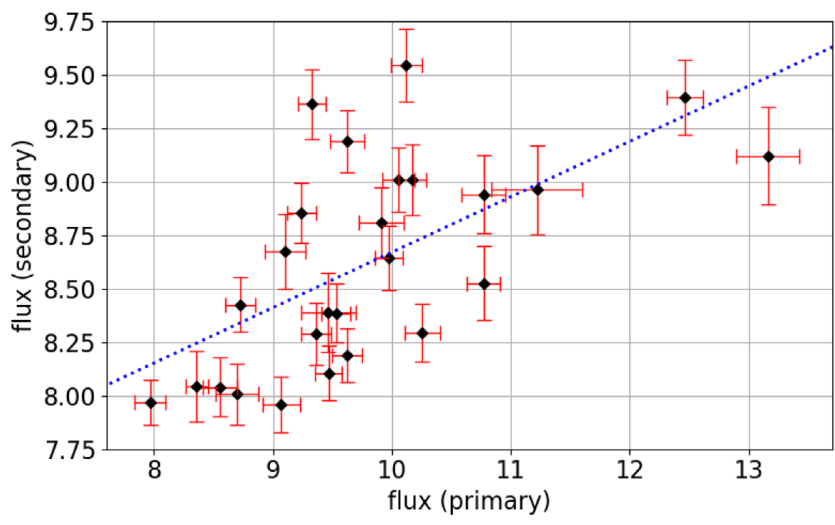

Figure 8. Comparison of the fluxes in the $\mathrm{Ca} I \mathrm{H} \mathrm{H}$ and $\mathrm{K}$ lines for both components. We use the $\mathrm{S} / \mathrm{N}$ ratio of each spectrum in the $\mathrm{Ca}$ II region to estimate the flux errors. Flux units are $\left(10^{-12} \mathrm{erg} \mathrm{cm}^{-2} \AA^{-1} \mathrm{~s}^{-1}\right)$.

this second period as due to this effect. In Fig. 7(b), we show the photometric observations phased with the period of $2030 \mathrm{~d}$.

To study the chromospheric activity of the system, we use the flux in the Ca II H and K lines. Since we have separated the spectra of both components, we should be able to distinguish whether the changes observed in the photometric measurements belong to one of the components or to both. The spectroscopic measurements also provide further information about the active regions present in

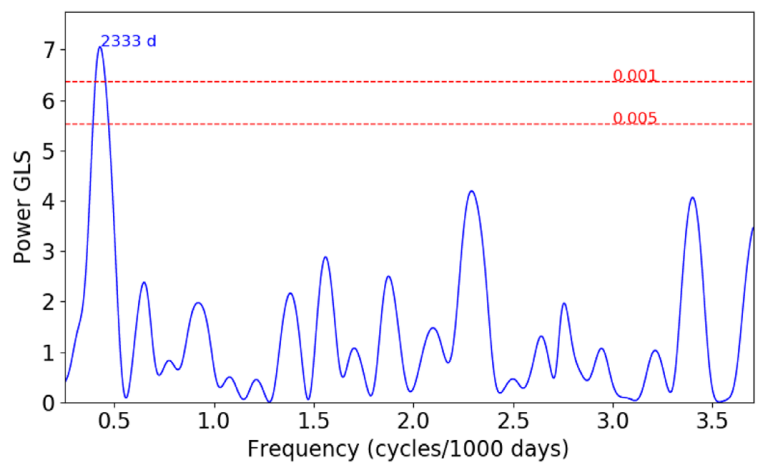

(a)

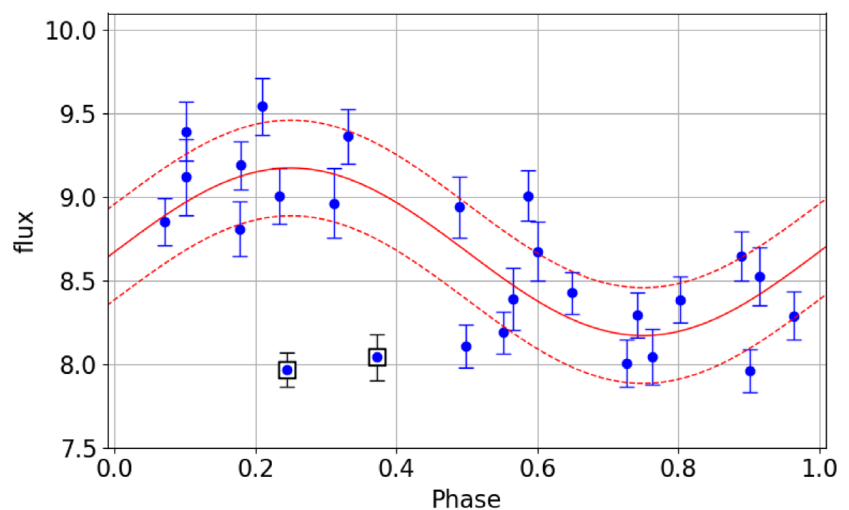

(b)

Figure 9. (a) GLS periodogram of the Ca II $\mathrm{H}$ and $\mathrm{K}$ flux for the secondary component; the most significant peak is indicated; the dashed lines indicate the FAP levels. (b) Phase curve for the $P=2333 \mathrm{~d}$ (the dashed lines represent $\pm \sigma$ deviations). We use the $\mathrm{S} / \mathrm{N}$ ratio of each spectrum in the $\mathrm{Ca}$ II region to estimate the error fluxes. The square points are the outliers mentioned in the text. The units are $\left(10^{-12} \operatorname{erg~cm}{ }^{-2} \AA^{-1} \mathrm{~s}^{-1}\right)$.

the stellar surfaces. We carefully examined the separated spectra and discarded the spectra exhibiting signs of reduction problems or transient events, such as flares. To do this, we compared the fluxes of the $\mathrm{H}$ and $\mathrm{K}$ lines in the individual spectra taken for each observation (see Section 2) and we discarded the pairs for which the difference was too large.

The fluxes in the $\mathrm{CaII} \mathrm{H}$ and $\mathrm{K}$ line-core emissions were integrated with a triangular profile of $1.09 \AA$, as is usually done to compute the Mount Wilson $S$-index. However, we did not normalize the flux by the continuum windows, since the continua are much weaker, and this would only introduce a large error factor. In Fig. 8, we plot the flux for both components of the system and the linear regression between them. It can be seen that both the fluxes are fairly correlated, with a Pearson correlation coefficient $r=0.62$.

We also computed the periodogram for the Ca II flux of each component. In Fig. 9, we show it for the secondary star. In this case, a period $P=2333 \pm 98$ with an FAP 0.0002 can be seen. There seem to be two outliers in the phase curve, which are responsible for most of the errors. They were removed from the calculations. On the other hand, we found no evidence of a period for the primary component.

From the separated CASLEO spectrum, we computed the $\log R_{\mathrm{HK}}^{\prime}$ (Noyes et al. 1984) indicator for each component. We obtained $\log R_{\mathrm{HK}}^{\prime}=-4.073$ for the secondary, the G-type star, and $\log R_{\mathrm{HK}}^{\prime}=-4.11$ for the primary, the K-type star. These values indicate that the secondary is the most active star of the 


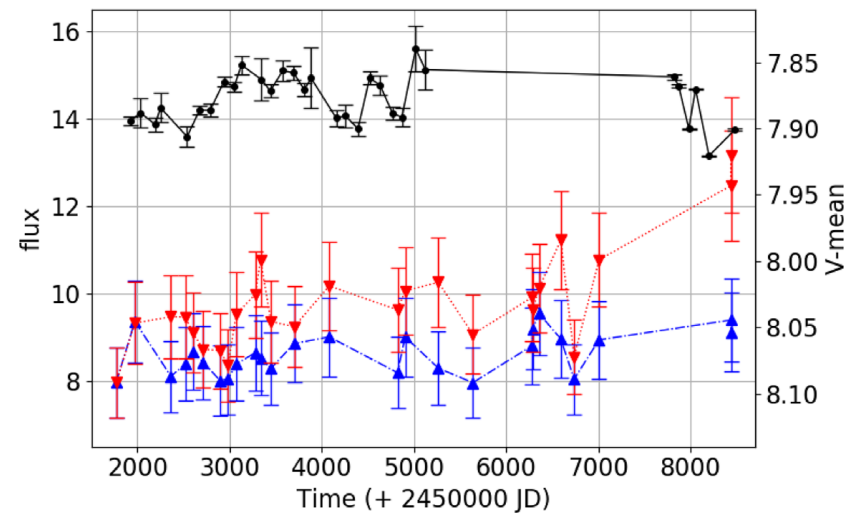

Figure 10. Mean magnitude (black solid lines), and Ca II $\mathrm{H}$ and $\mathrm{K}$ flux of the primary component (red dotted line) and of the secondary component (blue dashed line) as a function of time. The error bars correspond to a 10 per cent error in the line fluxes. Flux units are $\left(10^{-12} \mathrm{erg} \mathrm{cm}^{-2} \AA^{-1} \mathrm{~s}^{-1}\right)$.

system, and that a typical $\alpha \Omega$ dynamo could be responsible for the chromospheric cycle detected.

Finally, in Fig. 10, we plot the flux as a function of time for both components, together with the mean magnitude of the system. Note that the maximum for the secondary component took place in 2013 $(\mathrm{HJD}=2456355.5412)$.

\section{DISCUSSION}

The aim of this paper is to study the short-term variability and longterm variability of the spectroscopic binary system SZ Pictoris. For this purpose, medium-resolution Echelle spectra were collected at the CASLEO observatory, whereas photometric measurements in the $B V R I$ bands were obtained using the ORO, and the $V$-band data were taken from the ASAS data base. We found that the system exhibits high levels of chromospheric emission, as is expected for binary systems. We found no evidence of eclipses in the photometry. Therefore, the inclination angle must be low $(i \leq 63 \mathrm{deg})$ and the masses of the primary and secondary components cannot be lower than 1.54 and $0.82 \mathrm{M}_{\odot}$, respectively. These estimated masses agree with their spectral type.

We separated the spectra of both components, and we were able to determine accurate orbital parameters, in particular, an orbital period of $4.95 \mathrm{~d}$. The results are presented in Table 5 . In the different photometry data sets, we observed a modulation with half the orbital period, due to the ellipticity of the stars.

Studying the GLS periodogram of the long-term $V$ magnitude, we found that the system exhibits a possible periodic behaviour of $5.5 \mathrm{yr}$ (or $\sim 2030 \mathrm{~d}$ ). A cycle with a similar period can also be observed in the Ca II fluxes of the secondary component, the least active star of the system. The presence of this modulation in the two independent data sets reinforces the detection of the cycle. On the other hand, there is a phase difference between the cycles in the photometry and the Ca II flux, with the latter delayed by $554 \mathrm{~d}$, about $P / 4$. This time lag between photometric and magnetic variations has already been observed for stars of different spectral types (Díaz et al. 2007), although for cooler stars and with the opposite sense. It is also interesting that, although the Ca II flux in both stars is fairly correlated, only one of the components shows a cycle, something which is also different from what we found for the cooler system studied in Díaz et al. (2007).

Table 5. Spectra analysis summarized. Column 1 is heliocentric Julian date xJD = HJD - 2450000; columns 2 and 3 are RVs and Ca II flux for primary component, respectively; columns 4 and 5 are RVs and Ca II flux for secondary component, respectively.

\begin{tabular}{lrcrc}
\hline $\begin{array}{l}\mathrm{HJD}-2450000 \\
(\text { days })\end{array}$ & $\begin{array}{c}\mathrm{RV}(\text { primary }) \\
\left(\mathrm{km} \mathrm{s}^{-1}\right)\end{array}$ & $\begin{array}{c}f(\text { primary) } \\
\left(10^{-12} \mathrm{erg} \mathrm{cm}^{-2} \AA^{-1} \mathrm{~s}^{-1}\right)\end{array}$ & $\begin{array}{c}\mathrm{RV}(\mathrm{secondary}) \\
\left(\mathrm{km} \mathrm{s}^{-1}\right)\end{array}$ & $\begin{array}{c}f(\text { secondary }) \\
\left(10^{-12} \mathrm{erg} \mathrm{cm}^{-2} \AA^{-1} \mathrm{~s}^{-1}\right)\end{array}$ \\
\hline 1769.9198 & $13.4 \pm 3.8$ & $7.97 \pm 0.13$ & $-50.9 \pm 5.5$ & $7.97 \pm 0.10$ \\
1972.5502 & $32.6 \pm 2.9$ & $9.33 \pm 0.12$ & $-81.5 \pm 5.2$ & $9.36 \pm 0.16$ \\
2363.5186 & $36.7 \pm 3.9$ & $9.47 \pm 0.11$ & $-87.3 \pm 6.1$ & $8.11 \pm 0.13$ \\
2519.8542 & $-36.2 \pm 3.0$ & $9.46 \pm 0.23$ & $39.2 \pm 6.1$ & $8.39 \pm 0.19$ \\
2600.7692 & $40.1 \pm 2.2$ & $9.10 \pm 0.17$ & $-104.1 \pm 6.2$ & $8.68 \pm 0.18$ \\
2714.5270 & $44.7 \pm 2.8$ & $8.72 \pm 0.13$ & $-101.3 \pm 5.7$ & $8.43 \pm 0.13$ \\
2897.7720 & $42.4 \pm 2.5$ & $8.70 \pm 0.18$ & $-103.0 \pm 6.3$ & $8.01 \pm 0.14$ \\
2980.7187 & $-6.5 \pm 3.8$ & $8.36 \pm 0.09$ & $-13.8 \pm 5.3$ & $8.04 \pm 0.17$ \\
3073.5522 & $-55.7 \pm 3.1$ & $9.53 \pm 0.12$ & $88.1 \pm 5.6$ & $8.39 \pm 0.14$ \\
3276.8205 & $-50.0 \pm 4.8$ & $9.97 \pm 0.12$ & $78.8 \pm 5.8$ & $8.64 \pm 0.15$ \\
3335.7444 & $-58.3 \pm 2.4$ & $10.77 \pm 0.14$ & $85.1 \pm 7.8$ & $8.53 \pm 0.17$ \\
3448.5788 & $-10.2 \pm 5.3$ & $9.36 \pm 0.12$ & $2.0 \pm 5.2$ & $8.29 \pm 0.14$ \\
3699.7699 & $41.4 \pm 4.5$ & $9.24 \pm 0.12$ & $-103.1 \pm 5.4$ & $8.86 \pm 0.14$ \\
4080.7643 & $42.8 \pm 4.5$ & $10.17 \pm 0.11$ & $-101.5 \pm 5.4$ & $9.01 \pm 0.17$ \\
4820.6945 & $-55.1 \pm 3.9$ & $9.62 \pm 0.12$ & $82.6 \pm 5.5$ & $8.19 \pm 0.12$ \\
4903.5406 & $10.6 \pm 5.4$ & $10.06 \pm 0.13$ & $-45.1 \pm 5.1$ & $9.01 \pm 0.15$ \\
5263.5212 & $36.5 \pm 4.4$ & $10.26 \pm 0.15$ & $-85.2 \pm 5.7$ & $8.29 \pm 0.14$ \\
5637.5522 & $-60.4 \pm 4.6$ & $9.07 \pm 0.16$ & $81.3 \pm 5.9$ & $7.96 \pm 0.13$ \\
6281.7795 & $-47.0 \pm 4.5$ & $9.91 \pm 0.19$ & $70.9 \pm 5.9$ & $8.81 \pm 0.16$ \\
6284.6758 & $6.7 \pm 4.2$ & $9.62 \pm 0.14$ & $-48.7 \pm 5.2$ & $9.19 \pm 0.15$ \\
6355.5412 & $-61.7 \pm 4.5$ & $10.12 \pm 0.13$ & $83.0 \pm 5.3$ & $9.54 \pm 0.17$ \\
6591.8426 & $-11.4 \pm 4.3$ & $11.22 \pm 0.38$ & $-15.0 \pm 4.5$ & $8.96 \pm 0.21$ \\
6735.5367 & $-10.8 \pm 4.6$ & $8.55 \pm 0.14$ & $-6.5 \pm 4.9$ & $8.04 \pm 0.14$ \\
7006.8318 & $34.8 \pm 3.7$ & $10.77 \pm 0.18$ & $-97.6 \pm 5.8$ & $8.94 \pm 0.18$ \\
8435.7348 & $-16.0 \pm 2.0$ & $12.47 \pm 0.15$ & $6.2 \pm 8.4$ & $9.39 \pm 0.17$ \\
8436.6167 & $32.9 \pm 2.6$ & $13.16 \pm 0.26$ & $-84.1 \pm 8.7$ & $9.12 \pm 0.23$ \\
\hline & & & &
\end{tabular}


Therefore, the magnetospheres of the stars must be interacting. Vahia (1995) simulated the interaction of the magnetic field of binary stars, modelled as perfect dipoles surrounded by vacuum. They showed that it should be quite common to find one of the components located completely inside the magnetosphere of the companion. Zaqarashvili, Javakhishvili \& Belvedere (2002) described the mechanisms by which magnetic activity can be enhanced in interacting systems like this one, which would explain the high emission levels in the CaII lines. A similar effect was observed by Díaz et al. (2007) in another binary system.

The activity period we found places the system in the active branch of the $P_{\text {rot }}-P_{\text {cyc }}$ diagram found by Böhm-Vitense (2007) for solar-type stars, which is consistent with the level of activity of the stars under study.

\section{ACKNOWLEDGEMENTS}

This study is based on the data obtained at Complejo Astronómico El Leoncito, operated under agreement between the Consejo Nacional de Investigaciones Científicas y Técnicas de la República Argentina and the National Universities of La Plata, Córdoba, and San Juan.

\section{REFERENCES}

Andersen J., Nordström B., Olsen E., 1980, Information Bulletin on Varaible Stars, 1821

Baliunas S. L. et al., 1995, ApJ, 438, 269

Bell B. M., Hall D. S., Marcialis R. L., 1983, Information Bulletin on Variable Stars, 2272

Bilir S. et al., 2008, Astron. Nachr., 329, 835

Böhm-Vitense E., 2007, ApJ, 657, 486

Buccino A. P., Mauas P. J. D., 2009, A\&A, 495, 287

Buccino A. P., Díaz R. F., Luoni M. L., Abrevaya X. C., Mauas P. J. D., 2011, AJ, 141, 34

Buccino A. P., Petrucci R., Jofré E., Mauas P. J. D., 2014, ApJ, 781, L9

Cincunegui C., Mauas P. J. D., 2004, A\&A, 414, 699

Cincunegui C., Díaz R. F., Mauas P. J. D., 2007a, A\&A, 461, 1107

Cincunegui C., Díaz R. F., Mauas P. J. D., 2007b, A\&A, 469, 309

Cutispoto G., 1995, A\&AS, 111, 507

Cutispoto G., 1998a, A\&AS, 127, 207
Cutispoto G., 1998b, A\&A, 131, 321

Díaz R. F., González J. F., Cincunegui C., Mauas P. J. D., 2007, A\&A, 474, 345

Drake S. A., Simon T., Linsky J. L., 1989, ApJS, 71, 905

Flores M. G., Buccino A. P., Saffe C. E., Mauas P. J. D., 2017, MNRAS, 464, 4299

Flower P. J., 1996, ApJ, 469, 355

Gaia Collaboration, 2018, A\&A, 616, 22

González J. F., Levato H., 2006, A\&A, 448, 283

Gurzadyan G. A., 1997a, New Astron., 2, 31

Gurzadyan G., 1997b, MNRAS, 290, 607

Hall D. S., 1976, Astrophys. Space Sci. Libr., 60, 287

Hauck B., Mermilliod M., 1998, A\&AS, 129, 431

Henry T. J., Soderblom D. R., Donahue R. A., Baliunas S. L., 1996, AJ, 111,439

Horne J. H., Baliunas S. L., 1986, ApJ, 302, 757

Husser T.-O., Wende-von Berg S., Dreizler S., Homeier D., Reiners A., Barman T., Hauschildt P. H., 2013, A\&A, 553, A6

Ibañez R. V., Buccino A. P., Flores M., Martinez C. I., Maizel D., Messina S., Mauas P. J. D., 2019, MNRAS, 483, 1159

Metcalfe T. S. et al., 2013, ApJ, 763, L26

Noyes R. W., Hartmann L. W., Baliunas S. L., Duncan D. K., Vaughan A. H., 1984, ApJ, 279, 763

Pallavicini R., Randich S., Giampapa M. S., 1992, A\&A, 253, 185

Pojmanski G., 2002, Acta Astron., 52, 397

Press W. H., Teukolsky S. A., Vetterling W. T., Flannery B. P., 1992, Numerical Recipes in C. The Art of Scientific Computing, 2nd edn. Cambridge Univ. Press, Cambridge

Rocha-Pinto H. J., Maciel W. J., 1998, MNRAS, 298, 332

Rocha-Pinto H. J., Maciel W. J., Scalo J., Flynn C., 2000, A\&A, 358, 850

Roettenbacher R. M. et al., 2016, Nature, 533, 217

Schlegel D. J., Finkbeiner D. P., Davis M., 1998, ApJ, 500, 525

Schuster W. J., Nissen P. E., 1989, A\&A, 222, 69

Strassmeier K. G., Hall D. S., Fekel F. C., Scheck M., 1993, A\&AS, 100, 173

Turon C. et al., 1992, Bulletin d'Information du Centre de Donnees Stellaires, 41, 9

Vahia M. N., 1995, A\&A, 300, 158

Zaqarashvili T., Javakhishvili G., Belvedere G., 2002, ApJ, 579, 810

Zechmeister M., Kürster M., 2009, A\&A, 496, 577

This paper has been typeset from a $\mathrm{T}_{\mathrm{E}} \mathrm{X} / \mathrm{LT} \mathrm{E} \mathrm{X}$ file prepared by the author. 